Anna Plaksin

\title{
Repertoireüberschneidungen römischer und osteuropäischer Quellen
}

Ausgehend von der Beschäftigung mit den Chorbüchern der päpstlichen Kapelle, scheint die Themenstellung »Polyphonie in Polen, Schlesien und Böhmen« zunächst derart weit entfernt, dass sich nur schwer eine Brücke schlagen lässt. So stellte sich in diesem Zusammenhang die simple Frage: Wenn es sich bei der frühneuzeitlichen Vokalpolyphonie um eine gesamteuropäische Praxis handelte, warum lässt sich spontan so wenig >sinnvolles` über eine Verbindung dieser beiden lokalen Sphären sagen?

Dieser Ratlosigkeit sollte durch ein Experiment ${ }^{2}$ entgegengetreten werden, dessen Ergebnisse dieser Beitrag präsentiert. Im Zentrum steht die Frage, wie sich Repertoires und Überlieferungstraditionen verschiedener musikhistorischer Sphären miteinander vergleichen lassen. Ein erster Weg könnte in einer Studie konkordanter Werke bestehen. Doch ist die sich ergebende Schnittmenge derart gering, dass dies dem Gegenstand nicht gerecht würde. Stattdessen will dieser Beitrag vielmehr an einem Beispiel einen Vergleich der jeweiligen Repertoirezusammensetzung anhand quantitativ erfasster Katalogdaten liefern. Wie ein derartiges Vorgehen aussehen kann, soll in diesem Rahmen erprobt werden, um damit Perspektiven für weitere Forschungsarbeiten zu eröffnen.

Bei dem Versuch den Blick vom »römischen « Repertoire aus in Richtung Osteuropa auszuweiten, stößt man schon beinahe zwangsläufig auf ein erstes Hindernis profanster Art: Während die Forschungssituation zur päpstlichen Kapelle als komfortabel zu bezeichnen ist, scheitert man bereits an einer unübersichtlichen und teilweise auch unvollständigen Erfassung von handschriftlichen Quellen in polnischen und tschechischen Bibliotheken in den beiden großen Quellenverzeich-

I Dieser Aufsatz basiert auf der Arbeit an einem Dissertationsprojekt zum Repertoire der frühen Chorbücher des Fondo Cappella Sistina, dem auch die Daten zu den Handschriften entnommen sind.

2 Dem Versuch einer systematischen Repertoirestudie geschuldet, liegt der Fokus in diesem Beitrag auf der Erprobung der Möglichkeiten zur Kontextualisierung quantitativer Untersuchungsansätze. 
nissen, dem Census Catalogue ${ }^{3}$ und der RISM-Serie B/IV. Die Frage, wie sehr die bisherige Forschungsperspektive durch den Eisernen Vorhang getrübt war, drängt sich in diesem Zusammenhang auf. Dass hier offenbar tatsächlich ein blinder Fleck herrscht, der allerdings im Rahmen der politischen Entwicklungen der letzten 25 Jahre sich allmählich aufzulösen scheint, konnte im Rahmen des Symposiums und seiner Referate gezeigt werden. ${ }^{5}$ Von diesen Gedanken ausgehend zeigt sich, dass die Frage nach der >gefühlten Entfernung ‘ zwischen päpstlicher Kapelle und >osteuropäischer Peripherie< unter den aktuellen Vorrausetzungen neu gestellt werden sollte.

Die Ausgangsbasis ${ }^{6}$ dieser Untersuchung bildet die älteste Überlieferung polyphoner Ordinariumssätze der päpstlichen Kapelle; es handelt sich um die Handschriften Cappella Sistina I4, 5I, 23, 35, 4I, 49, 63 und I97? In ihnen sind hauptsächlich 9I größtenteils vollständige Ordinariumszyklen und einzelne Messteile überliefert. Weitere Gattungen geistlicher Polyphonie bilden nur einen kleinen Teil, da diese zumeist in eigenen Handschriftensammlungen einen Platz finden.

Festzuhalten ist, dass sich die Konkordanzen dieses Repertoires über den gesamten europäischen Raum erstrecken, wobei allerdings nur ein marginaler Teil von sechs Messen auch in Handschriften böhmischer, schlesischer oder polnischer Herkunft ${ }^{8}$ vorhanden ist:

3 Charles Hamm, Herbert Kellmann (Hrsg.), Census Catalogue of Manuscript Sources of Polyphonic Music I400-I550, 5 Bde., Neuhausen-Stuttgart I979-I988 (Renaissance Manuscript Studies I).

4 Kurt von Fischer, Max Lütolf, Handschriften mit mehrstimmiger Musik des I4., I5. und I6 Jahrhunderts. Mehrstimmige Musik in italienischen, polnischen und tschechischen Quellen des 44 Jahrhunderts; mehrstimmige Stücke in Handschriften aller Länder aus der Zeit um I400-I425/30; organale Sätze im älteren Stil und mehrstimmige Stücke in Choralhandschriften des Is. und I6. Jahrhunderts, 2 Bde., Kassel u.a. 1972; Nanie Bridgman, Manuscrits de musique polyphonique. XVe et XVIe siècles, Italie, München I99I.

5 Besonders sei hier auf den Beitrag von Christiane Wiesenfeldt verwiesen.

6 Vgl. Richard Sherr, The Papal Chapel ca. I492-I5I3 and its Polyphonic Sources, PhDiss., Princeton I975; Jeffery J. Dean, The Scribes of the Sistine Chapel I50I-I527, PhDiss., Chicago I984; Albert Roth, Studien zum frühen Repertoire der Päpstlichen Kapelle unter dem Pontifikat Sixtus' IV. (I47I-I484). Die Chorbücher I4 und sI des Fondo Cappella Sistina der Biblioteca Apostolica Vaticana, Vatikanstadt I99I (Capellae Apostolicae Sixtinaeque Collectanea Acta Monumenta I) und Nanie Bridgman, Manuscrits de musique polyphonique (wie Anm. 4). Die verwendete Aufstellung der Werkkonkordanzen basiert auf der genannten Literatur und den kritischen Berichten vorhandener wissenschaftlichen Editionen der Werke.

7 I-Rvat: Capp. Sist. I4, Capp. Sist. 23, Capp. Sist. 35, Capp. Sist. 4I, Capp. Sist. 49, Capp. Sist. 5I, Capp. Sist. 63, Capp. Sist. I97.

8 Der Einfachheit halber soll diese Gruppe im Folgenden vereinfacht als >osteuropäische $<$ Handschriften bezeichnet werden. 
- CZ-HK II A 7 (Codex Speciálník)

- Missa l'ami baudichon (Josquin) in CS 23

- Missa de Franza (Basiron) in CS 35

- Missa O Venus bant (Gaspar) in CS 5 I

- PL-Wu RM 5892

- Missa O Venus bant (Gaspar) in CS $5 \mathrm{I}$

- Missa Quant j'ay au cueur (Isaac) in CS 35

- PL-Pu 7022 (Lwow-Fragment)

- Missa Spiritus almus (de Domarto) in CS I4

- Missa l'ami baudichon (Josquin) in CS 23

- Missa l'homme armé sexti toni (Josquin) in CS 4I

Anhand einer genaueren Übersicht der Handschriften jener Provenienz zeigt sich, dass auch in umgekehrter Richtung die Unterschiede überwiegen. So erscheint die überwiegend anonyme Überlieferung innerhalb dieser Handschriften hierbei besonders auffällig.

- 40 Handschriften

- Herkunft:

- polnisch

- tschechisch

- I45O-I55O
- 7 Fragmente

- 3 Stimmbücher

- 4 Textquellen mit polyphonen Hinzufügungen

- 4 Sammelhandschriften mit meist polyphonem Repertoire

- 22 Hs. mit meist einstimmiger Kirchenmusik

Tabelle I: >Osteuropäische< Handschriften: Übersicht ${ }^{9}$

Der Großteil der vierzig so eingegrenzten Handschriften überliefert in erster Linie einstimmige Kirchenmusik oder Texte, außerdem ist ein erheblicher Teil nur fragmentarisch erhalten. In ihnen nimmt die polyphone Musik lediglich eine untergeordnete Stellung ein. Selbst der unter den Konkordanzen vertretene Codex Speciálník ${ }^{10}$ vereint kein rein polyphones Repertoire. Das völlige Fehlen von Agnus Dei-Vertonungen weist zudem vorrangig auf den Gebrauch im Rahmen der hussitischen Liturgie hin. ${ }^{11}$ Dem gegenüber ist am Lwow-Fragment ein italie-

9 Diese Aufstellung basiert auf dem Register des Census Catalogue Bd. 5, S. 274f. und 288 (wie Anm. 3). Für die Beschreibungen der Handschriften, vgl. Census Catalogue und die Manuscript Database des Digital Image Archive of Medieval Music (DIAMM), abrufbar unter http://www.diamm.ac.uk/ms-database/(I3.0I.20I4).

IO CZ-HK: II A 7.

I I Vgl. Laura Kozachek, Thomas M. Höpfner(Übs.), »Codex Speciálník «, in: Die Musik in Geschichte und Gegenwart, 2. Aufl., hrsg. von Ludwig Fischer, Sachteil Bd. 8, Kassel u.a. I998, Sp. I682-I684. 
nischer Einfluss deutlich erkennbar, auf den auch Mirosław Perz hinweist. ${ }^{12}$ So ist etwa Domartos Missa »Spiritus almus « nur in italienischen Quellen zu finden. ${ }^{13}$

Um aber wiederum im Vergleich auf die Messen in den Handschriften der Cappella Sistina zu blicken, ist über Umwege anhand der Komponistenzuordnung der Werke eine Annäherung an die Zusammensetzung des päpstlichen Repertoires möglich. Betrachtet man die Biographien der vertretenen Komponisten ${ }^{14}$ auf ihre Beziehung zur päpstlichen Kapelle hin, ergibt sich für die überlieferten Werke folgendes Bild:

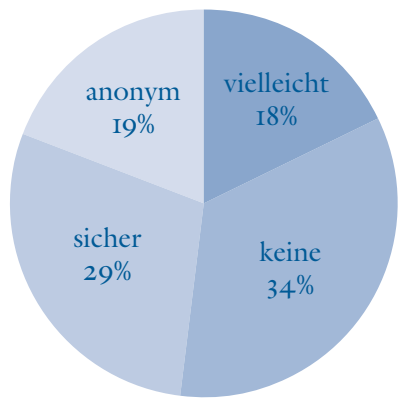

\begin{tabular}{lc} 
Verbindung & Anzahl Werke \\
\hline Anonym & I9 \\
Sicher & 29 \\
Vielleicht & I 8 \\
Keine & 34 \\
\hline Summe & IOO
\end{tabular}

Abbildung I: Werke nach Beziehung der Komponisten

Im Gegensatz zu den überwiegend anonymen Werken in den osteuropäischen Handschriften sind hier lediglich ca. I9\% der Werke anonym überliefert. 29\% der Werke stammen von Komponisten, die der päpstlichen Kapelle angehörten, den deutlich größten Teil nehmen hier die Kompositionen von Josquin ein. 34\% der Werke stammen von Komponisten, die nicht Mitglied und wohl auch, wie z.B. Philippe Basiron, niemals in Italien waren. Über die Komponisten der übrigen I8\% ist entweder beinahe nichts Biographisches bekannt, wie z.B. bei Firminus Caron, oder es sind Romreisen überliefert, wie im Falle von Loyset Compère. Es kann also davon ausgegangen werden, dass sich im Repertoire eigene Werke wie auch fremdes Repertoire zumindest die Waage halten.

Aber nicht nur das päpstliche Repertoire zeichnet sich durch seine internationale Vernetzung aus. Es lässt sich unter anderem gerade in den osteuropäischen

I Vgl. Mirosław Perz, »The Lvov Fragments. A Source for Works by Dufay, Josquin, Petrus de Domarto and Petrus de Grudencz in I5th-Century Poland «, in: Tijdschrift van de Vereniging voor Nederlandse Muziekgeschiedenis 36 (I986), 26-5I, S. 39 f.

I3 Vgl. Perz, The Lvov Fragments (wie Anm. I2) S. 34.

I4 Es sind im Repertoire Zuschreibungen an 33 Komponisten vertreten, von denen lediglich acht (24\%) eine sichere Verbindung zur Päpstlichen Kapelle nachgewiesen werden kann, I6 Komponisten $(49 \%)$ kann keine Verbindung nachgewiesen werden, neun Komponisten (27\%) kann vielleicht eine Verbindung nachgewiesen werden. 
Handschriften, in denen sich die benannten Konkordanzen befinden, ein Kreis ausmachen, der erheblich zur Verbreitung polyphoner Musik beigetragen hat und von Reinhard Strohm als »inner network ${ }^{15}$ bezeichnet wird. Durch dieses Netzwerk, das laut Strohm bisher nur unzureichend bekannt ist, verbreitete sich polyphones Repertoire von den Trienter Kodizes über Quellen wie den Apel-Kodex ${ }^{16}$ oder den Leopold-Kodex ${ }^{17}$ hin zu dem bereits erwähnten Codex Speciálník, den Handschriften in Warschau und Posen und auch dem Strahov-Kodex. ${ }^{18}$ Über diese Verbindung, besonders über die Trienter Handschriften, lässt sich auch eine Verbindung zum Repertoire der päpstlichen Kapelle ziehen.

Wie nun aber ist diese, gleichwohl vorläufige, Situation zu bewerten? Im Rückgriff auf das Bild des Eisernen Vorhangs ließe sich nach den musikkulturellen Grenzen im Europa um I500 fragen. Ebenso naheliegend ist auch der Verweis auf die immer wieder genutzten Kategorien >Peripherie $<$ und $>$ Zentrum $<$. Nur wie soll eine derartige Zuordnung herzustellen sein?

Bezogen auf das Repertoire haben Böhmen, Schlesien und Polen nicht viel mit den Chorbüchern der Sixtinischen Kapelle zu tun. Dennoch zeigt das von Strohm so benannte »inner network «, ${ }^{19}$ dass hier keineswegs von einer Isoliertheit zu sprechen ist. Vielmehr können die genannten Quellen gemeinsam mit anderen als Indiz für einen hohen Grad an Vernetzung im habsburgischen Raum gewertet werden. Gleichwohl ist zudem zu bedenken, dass hier sehr viel mehr Quellen polyphoner Musikausübung bekannt sind, als es in anderen Regionen des Kaiserreichs der Fall ist. Beispielsweise lassen sich in diesem Punkt die Städte am Rhein zu dieser Zeit geradezu als weiße Flecken auf der musikhistorischen Landschaft bezeichnen, da zumeist nur fragmentarische Funde bekannt sind. ${ }^{20}$ Die gesuchte kulturelle Grenze lässt sich, so scheint es, nicht so einfach ziehen, wie es in den politischen Verhältnissen des 20. Jahrhunderts der Fall war, sondern zeigt sich sehr viel differenzierter.

Ein wesentlicher Unterschied zeigt sich daneben in einer fundamental abweichenden Situation der Überlieferung. Betrachtet man die in den >osteuropäischen< Handschriften vertretenen Gattungen, lässt sich folgende Verteilung beobachten:

I 5 Reinhard Strohm, The Rise of the European Music 1380-I500, Cambridge 2005, S. 5IIf.

I6 D-LEu: MS I494.

I 7 D-Mbs: Mus. MS 3 I5 (olim: MaiM 42; Chorbuch des Nikolaus Leopold).

I 8 CZ-Ps: MS D.G.IV.47.

I9 Vgl. Strohm, The Rise (wie Anm. I5), ebd.

20 Vgl. Laurenz Lütteken, »Politische Zentren als musikalische Peripherie? Probleme einer musikhistorischen Topographie im deutschen Nordwesten des I5. und I6. Jahrhunderts «, in: Das Erzbistum Köln in der Musikgeschichte des I5. und I6. Jabrhunderts, hrsg. von Klaus Pietschmann, Kassel 2008 S. 6I-75. 


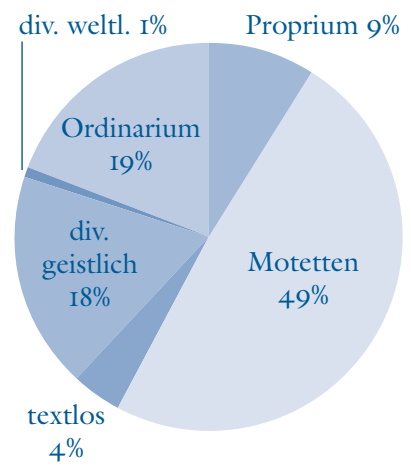

\begin{tabular}{lr} 
Gattung & Anzahl \\
\hline Ordinariumsteile und -zyklen & 252 \\
Propriumsteile und -zyklen & I I 8 \\
Motetten & 647 \\
Div. geistliche Gattungen & 234 \\
Div. weltliche Gattungen & I 5 \\
Textlose Stücke & 50 \\
\hline Summe & I3 I6
\end{tabular}

Abbildung 2: Repertoireübersicht nach Gattungen

Auf den ersten Blick sticht hierbei das Übergewicht an Motetten ins Auge, die beinahe 50\% des Gesamtrepertoires ausmachen. Daneben finden sich alle Gattungen geistlicher Musik sowie auch ein kleiner Anteil weltlicher Musik. Da der Fokus bisher auf den Ordinariumsvertonungen lag, sollen diese auch hier genauere Betrachtung finden:

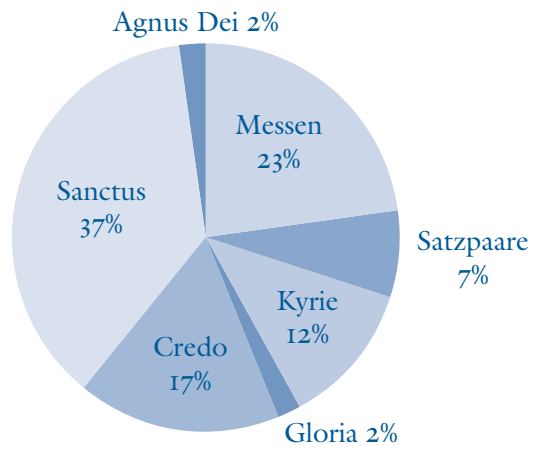

\begin{tabular}{lr} 
Gattung & Anzahl \\
\hline Messen & 58 \\
Satzpaare & I 8 \\
Kyrie & 29 \\
Gloria & 6 \\
Credo & 43 \\
Sanctus & 92 \\
Agnus Dei & 6 \\
\hline Summe & 252
\end{tabular}

Abbildung 3: Ordinariumsrepertoire im Detail

Im Gegensatz zum päpstlichen Repertoire machen hier ganze bzw. unvollständige Zyklen $^{21}$ nur 23\% des Repertoires aus, gemeinsam mit den Satzpaaren sind es $30 \%$. Der Großteil sind somit Einzelsätze, wobei sich auch hier wieder in der weit unterdurchschnittlichen Anzahl von Agnus Dei-Vertonungen eine konfessionelle Spielart zeigt.

2 I Hierzu zählen auch Zyklen, die nur drei oder vier Teile beinhalten. 
Erweitert man nun - im Vergleich - den Blick auf das päpstliche Repertoire um jene Chorbücher im gewählten Zeitraum, die keine Messvertonungen überliefern, zeigt sich in einer chronologischen Übersicht ${ }^{22}$ folgendes Bild:

\begin{tabular}{|c|c|c|c|}
\hline Signatur & Datierung & Inhalt & Typ \\
\hline CS I4 & $\mathrm{I} 472-\mathrm{I} 48 \mathrm{I}$ & Messen, Messteile & Schenkung \\
\hline CS $5 \mathrm{I}$ & I472-I49I & Messen, Messteile & Schenkung \\
\hline $\mathrm{CS}_{35}$ & I487-I499 (ca.) & Messen, Messteile, Motetten & Gemischt \\
\hline CS 63 & I $480-\mathrm{I} 507$ (ca.) & Messen, Magnificats, Motetten & Gemischt \\
\hline CS 197 & I492-I495 (ca.) & Messe & Messhandschrift \\
\hline $\mathrm{CS}_{4 \mathrm{I}}$ & $\mathrm{I} 482-\mathrm{I} 507$ (ca.) & Messen, Messteile & Messhandschrift \\
\hline CS I5 & I492-I 5OI (ca.) & Magnificats, Hymnen, Motetten & Andere Gattungen \\
\hline CS 49 & I $492-\mathrm{I} 5 \mathrm{O} 4$ (ca.) & Messen, Motette & Gemischt \\
\hline CS 23 & I $492-$ I 509 (ca.) & Messen, Messteile & Messhandschrift \\
\hline $\mathrm{CS}_{44}$ & I $503-\mathrm{I} 5 \mathrm{I} 2$ (ca.) & Magnificats, Motetten, Benedictus & Andere Gattungen \\
\hline $\mathrm{CS}_{42}$ & I $504-\mathrm{I} 5 \mathrm{I} 2$ (ca.) & Passionen, Motetten & Andere Gattungen \\
\hline $\mathrm{CS}_{46}$ & I5O8-I 527 (ca.) & Motetten & Andere Gattungen \\
\hline
\end{tabular}

Tabelle 2: Päpstliche Chorbücher bis $15 \mathrm{IO},{ }^{23}$ chronologisch geordnet.

Anhand der vorgenommenen Typisierung nach vertretenen Gattungen des Repertoires, lässt sich eine zeitliche Entwicklung nachzeichnen, die Phasen von Repertoirewachstum und -konsolidierung im Rahmen der Reform der päpstlichen Kapelle entsprechen. Es lassen sich hierbei drei Stadien umreißen:

I. Die Begründung eines Repertoires mit den beiden vermutlich neapolitanischen Chorbüchern I4 und 5I, deren Repertoire teilweise genutzt wurde, teilweise aber auch nicht.

2. Der Beginn der eigenen Repertoiresammlung und Kanonisierung, hauptsächlich mit Ordinariumskompositionen.

3. Mit der Konsolidierung eines Grundrepertoires geht eine verstärkte Sammlung anderer geistlicher Gattungen einher.

22 Die Datierungen wurden der Literatur entnommen, vgl. hierzu Dean, Scribes (wie Anm. 6); Roth, Studien (wie Anm. 6) und Sherr, Papal Chapel (wie Anm. 6) . Außer im Falle der beiden Schenkungen handelt es sich bei den Chorbüchern um Sammlungen von Einzellagen, die der Übersichtlichkeit halber in dieser Aufstellung nicht einzeln aufgeführt sind, aber in den Datierungsangaben berücksichtigt wurden. Die Anordnung in der Aufstellung basiert, im Sinne einer Annäherung, auf den Mittelwerten der Zeiträume.

23 Hinzu kommen I-Rvat: Capp. Sist. I5, Capp. Sist. 42, Capp. Sist. 44, Capp. Sist. 46. 
Gerade die Möglichkeit, solche Repertoireentwicklungen ${ }^{24}$ im Überblick untersuchen zu können, demonstriert den besonderen >statistischen Glücksfall , den die päpstlichen Chorbücher darstellen. Bezogen auf die Ordinariumsvertonungen, zeigt sich, dass diese offenbar integraler Bestandteil des Repertoires der päpstlichen Kapelle waren, insbesondere die Sammlungen vollständiger Messzyklen. Inwiefern hierbei auch bereits künstlerische oder pragmatische Gründe eine Rolle spielen, ist noch weiter auszuführen.

Im Gegensatz hierzu tritt der abweichende Grundcharakter der osteuropäischen Handschriften besonders deutlich hervor, bei denen es sich zumeist um Quellen mit einem starken usuellen Charakter handelt. Ihr Repertoire scheint darauf angelegt zu sein, die Liturgie möglichst praktisch verschiedenen Gegebenheiten anpassen zu können, indem ein vielseitiges Repertoire mögliche Versatzstücke in einem Baukastenprinzip bieten kann.

Letztendlich zeigt sich in dem Versuch einer solchen geographischen Rundumschau, dass sich die Stellung kunstvoller Vokalpolyphonie nur adäquat fassen lässt, wenn sie nicht nur in ihrer Verbreitung, sondern gleichfalls auch im Kontext ihrer >Alternativen< gesehen wird. Während im päpstlichen Repertoire - wie die bisherige Forschung gezeigt hat - verschiedene subtile Mechanismen der Repertoiregestaltung ineinandergreifen, offenbart sich die Stellung einer derartigen musikalischen Gestaltung der Liturgie weniger in ihrer isolierten Betrachtung, sondern eben auch in einer umfassenden und auch stilübergreifenden Repertoiregenese. Des Weiteren wird deutlich, dass sich auf der Basis einer quantitativ orientierten Betrachtung Kontextualisierungen herausarbeiten lassen, die einen Vergleich ermöglichen, der über die Nennung bloßer Häufigkeiten hinausgeht. Mit einem derartigen Blick wäre auch die Frage nach kulturellen Grenzverläufen, Zentren und Peripherien neu zu bewerten.

24 Zur Kanonisierungspraxis der Päpstlichen Kapelle siehe Klaus Pietschmann, Kirchenmusik zwischen Tradition und Reform, Vatikanstadt 2007 (Capellae Apostolicae Sixtinaeque Collectanea Acta Monumenta II), S. I78-I87, besonders S. I80f. 